ISSN: $2675-469 \mathrm{X}$

Vol. 02 - n 03 - ano 2021

Editora Acadêmica Periodicojs

\title{
APPLICATION OF SEISMIC DILATOMETER FOR CEMENTATION ANALYSIS IN RESIDUAL GNEISS \\ SOIL
}

Fábio Krueger da Silva ${ }^{1}$

Fernanda Simoni Schuch ${ }^{2}$

Resumo: Os solos residuais têm diferenças são decorrentes dos propriedades e comportamento diferentes tipos de rocha matriz, distintos de solos sedimentares. mecanismos de decomposição Aspectos relacionados aos pro- envolvidos no processo e ações cessos de origem e formação antrópicas. Este artigo avaliou a impactam diretamento nestas mudanças do grau de cimentação particularidades geotécnicas. Um em horizontes de solo residual das propriedades características com aplicação do Dilatômetro deste material é a cimentação. de Marchetti Sísmico (SDMT). A cimentação é uma resistência Realizou-se a pesquisa num solo adicional que ocorre no esquele- residual de gnaisse do Estado de to sólido decorrente das ações do Santa Catarina. A avaliação do intemperismo sobre estes mate- grau de cimentação foi baseada riais. Num perfil de solo residu- nas relações obtidas com o Go al ocorrem diferentes níveis de (módulo cisalhante à pequenas cimentação entre os grãos, estas deformações) e outros índices 1 Engenheiro, Doutor em Infraestrutura Viária, Professor do Deparatamennto Acadêmico da Construção Civil do Instituto Federal de Santa Catarina, Florianópolis, Brasil, 2 Engenheira Doutora em Cadastro Técnico Multifnalitário e Gestão Territorial, Professora do Deparatamennto Acadêmico da Construção Civil do Instituto Federal de Santa Catarina, Florianópolis, Brasil

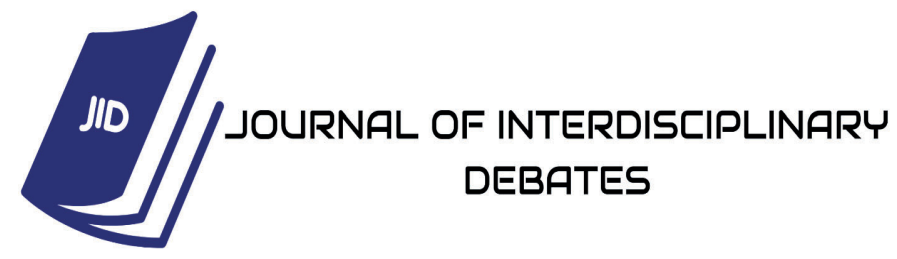


geotécnicos de campo. Os resultados mostram que, a interpretação das correlações a partir dos resultados obtidos pelos ensaios SPT (Ensaio de Penetração Padronizado), CPT (Ensaio de Penetração de Cone) e SDMT, permitem identificar limites de camadas com distintos graus de cimentação em solos residuais de gnaisse. No presente trabalho foram identificados os limites entre as camadas saprolíticas e as lateríticas e, por fim, concluindo-se que o solo residual estudado apresenta um baixo de nível de cimentação entre as partículas.

Palavras-chave: Cimentação, Solos residuais, Ensaio SDMT.

\begin{abstract}
Residual soils have different properties and behavior from sedimentary soils. Aspects related to the processes of origin and formation directly impact
\end{abstract}

these geotechnical particularities. One of the characteristic properties of this material is cementation. Cementation is an additional resistance that occurs in the soil skeleton due to weathering actions on these materials. In a residual soil profile, different cementation levels occur between particles, these differences are due to different types of matrix rock, decomposition mechanis$\mathrm{ms}$ involved in the process and anthropic actions. This article evaluated changes in the cementation tax in a residual soil using the Marchetti Seismic Dilatometer (SDMT). The research was carried out in a residual gneiss soil in Santa Catarina's State. The evaluation of the cementation tax was based on the relationships obtained with the Go (shear modulus with small strain) and other field geotechnical index. The results show that the

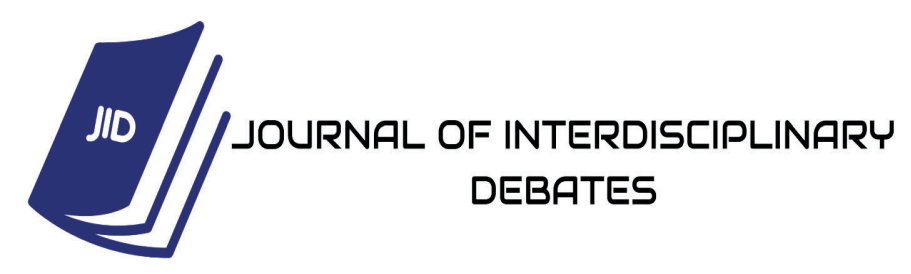


ISSN: 2675-469X

Vol. 02 - n 03 - ano 2021

Editora Acadêmica Periodicojs

interpretation of the correlations

from the results obtained with

SPT (Standardized Penetration

Test), CPT (Cone Penetration

Test) and SDMT tests, allow the

identification of layer boundaries

with different taxes of cementa-

tion in the residual gneiss soil.

In the present work, the limits

between the saprolitic and the la-

teritic layers were identified and,

it was concluded that, the studied

residual soil presents a low level

of cementation between its particles.

Keywords: Cementation, Residual Soil, SDMT test

\section{Introdução}

Os ensaios de campo são essenciais nas classificações, definições das camadas do solo, determinação de nível de água, estimativa de proprie- dades geotécnicas no estado natural do solo e, em alguns casos, coleta de material para análise. Estes ensaios garantem a rapidez na execução e determinação dos resultados, alto potencial de repetibilidade e avaliação de uma grande área com baixo custo.

Devido às particularidades que envolvem a macro e microestrutura de solos residuais e às limitações dos tradicionais métodos de estimativa, a partir de ensaios de campo, buscou-se nesta pesquisa verificar a ocorrência de cimentações atráves de correlações obtidas em investigação geofísica com o ensaio SDMT (Dilatômetro de Marchetti Sísmico).

Os métodos geofísicos tem conquistado espaço na geotecnia para explorações de caráter subsuperficial. Os testes são geralmente de caráter não destrutivos e podem ser re-

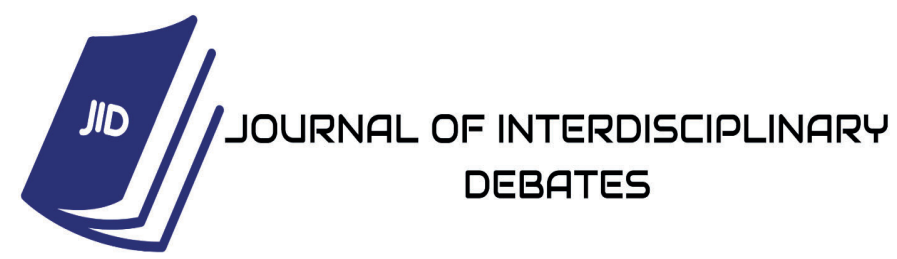


ISSN: 2675-469X

Vol. 02 - n 03 - ano 2021

Editora Acadêmica Periodicojs

alizados a partir da superfície do terreno. Particularmente, nesta pesquisa trata-se sobre o ensaio sísmico adaptado aos ensaios de campo convencionais. A tendência atual de desenvolvimento de novos equipamentos está na direção de se combinar diferentes técnicas, como o ensaio do cone, aliando a robustez do equipamento a medidas adicionais provenientes de outros ensaios. Por exemplo, o SCPTU (Piezocone Sismico) que fornece as variáveis usuais obtidas no CPT, contudo, adiciona informações importantes sobre a pressão neutra e o módulo sísmico do solo num mesmo equipamento. Aos poucos estas técnicas vêm ganhando espaço no Brasil para aplicações em engenharia civil e geotecnia ambiental (Schnaid, 2000).

\section{Revisão da Literatura}

\section{Solos residuais}

Dependendo do grau de alteração sofrido, alguns solos não apresentam nenhum resquício da rocha de origem, enquanto outros são fortemente influenciados pelas estruturas reliquiares da rocha mãe. Tais estruturas reliquiares deixam marcas nos solos originados através de ligações por cimentação ou ligações já rompidas. Ou ainda, marcas de fissuras e falhas que já ocorriam na rocha de origem (Mayne \& Brown, 2003).

Nas camadas que formam um perfil intemperizado pode-se encontrar desde rocha sã, rocha alterada, solo de alteração (solo residual jovem ou saprolítico) a uma camada tão alterada que já não reproduz características da rocha de origem (solo residual maduro ou laterítico). Normalmente, no topo do perfil

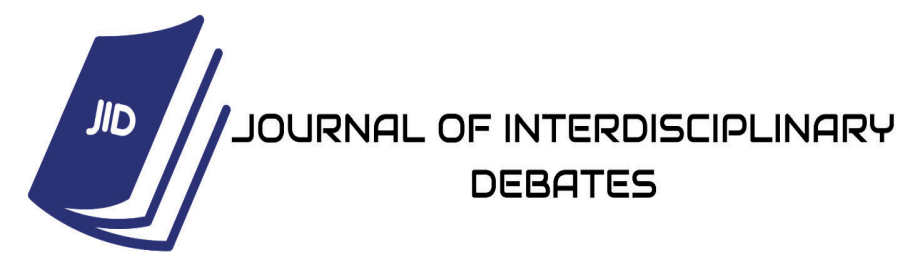


ISSN: 2675-469X

Vol. 02 - n 03 - ano 2021

Editora Acadêmica Periodicojs

deve existir uma camada de solo transportado (por exemplo, colúvio) que pode dificultar a identificação do verdadeiro solo residual. É usual estratificar o perfil intemperizado de um solo residual por horizontes. O horizonte I, localizado na superfície do solo, é caracterizado pela incidência de um solo transportado ou orgânico. O horizonte II é a camada conhecida por solo laterítico. Sua formação ocorre em locais de clima quente e úmido sendo caracterizado por possuírem alta permeabilidade e, normalmente apresentarem uma estrutura bem forte devido às ligações existentes provocadas pelo processo de laterização. O processo de laterização se refere à livixiação de minerais e, subsequente incremento de óxidos e hidróxidos de ferro e alumínio, que por meio da oxidação, acabam por enrijecer a estrutura do solo. (Viana da Fon- seca et al. 2006).

O horizonte III (solo saprolítico) pode apresentar um alto nível de heterogeniedade tanto na vertical como na horizontal, bem como um complexo arranjo estrutural no qual retêm informações da rocha de origem. A textura e a mineralogia podem variar muito nesta camada devido ao grau de alteração e lixiviação sofrido pelo solo. O horizonte IV é chamado de saprólito uma camada de transição entre o solo e a rocha. $\mathrm{O}$ horizonte $\mathrm{V}$ é uma camada de rocha altamente intemperizada. Em regiões tropicais a ação do intemperismo frenquentemente pode deixar estes dois últimos horizontes muito delgados ou até mesmo inexistentes. Neste local a transição entre o solo saprolítico e a rocha alterada ocorre em poucos centímetros (Viana da Fonseca et al. 2006).

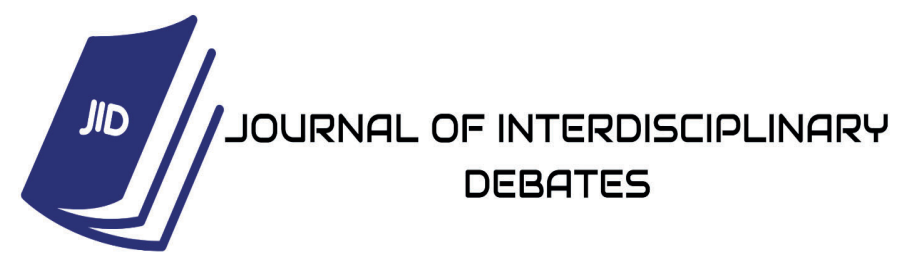


Vol. 02 - n 03 - ano 2021

Editora Acadêmica Periodicojs

Cimentação em solos residuais

Atualmente, este termo tem sido associado ao que é possível observar nos solos ao olho nu, por exemplo, planos de fraqueza, dobras, descontinuidades e vazios. Estes aspectos geotécnicos ficam mais bem adaptados utilizando-se o termo macroestrutura. Os estudos mais recentes ficam concentrados em compreender a maneira como as partículas do solo se encaixam, ou melhor, como é o arranjo estrutural entres os grãos do solo (Mitchell, 1993; Leroueil \& Vaughan, 1990 e Maccarini, 1987).

Este arranjo estrutural é que forma o esqueleto sólido do solo. Em alguns tipos de solos, este arranjo conta ainda com uma parcela de ligação entre as partículas formando solos altamente estruturados. Para este tipo de estrutura que não é possível ob- servar a olho nu dá-se o nome de microestrutura (Wesley, 2010).

A microestrutura dos solos residuais tem forte influência no seu comportamento mecânico, da mesma forma que o índice de vazios inicial e a história de tensões refletem nas propriedades dos solos sedimentares. A microestrutura do solo residual tem uma componente extra de resistência chamada de cimentação. Ela pode ser entendida como uma contribuição adicional na união que ocorre nos contatos grão a grão. É oriunda da época que o material original era uma rocha sã. Esta contribuição de resistência nos solos residuais é anisotrópica, com diferentes graus de cimentação ao longo do perfil e, também nas camadas horizontais, sendo resultados de processos físicos, químicos e biológicos que o solo sofre durante sua formação (Viana da Fonseca

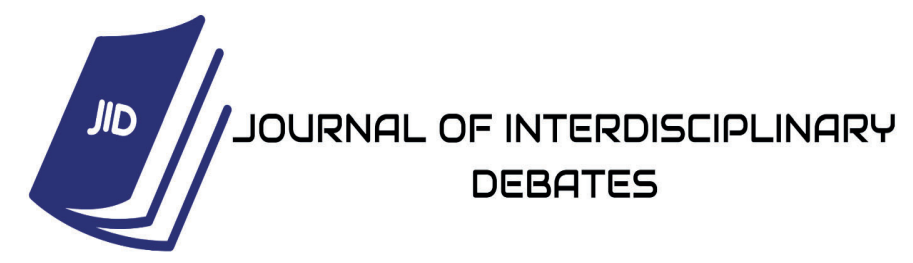


ISSN: 2675-469X

Vol. 02 - n 03 - ano 2021

Editora Acadêmica Periodicojs

et al. 2006).

Dessa forma, entende-se que os graus de cimentação são diferentes em horizontes lateríticos e saprolíticos de um solo residual. A identificação e quantificação de ligações cimentadas fracas ou fortes são importantes no entendimento do comportamento mecânico do material. A cimentação afeta muito a relação tensão x deformação. Porém, a determinação laboratorial desta parcela natural de resistência não é tarefa fácil porque nas amostragens convencionais, uma parte da cimentação pode ser destruída pelo simples desconfinamento da amostra, e/ou ainda, na preparação dos corpos de prova e dos níveis de tensões aplicadas nos ensaios. A estimativa através de ensaios de campo convencionais também tem uma componente de dificuldade devido a desestruturação provocada pela cravação das sondas nos solos residuais.

Assim, esta pesquisa identifica zonas com diferentes níveis de cimentação, através de ensaios sísmicos (Krueger, 2015).

\section{Ensaio Dilatômetro de Mar- chetti Sísmico - SDMT}

No caso do Dilatômetro sísmico (SDMT) foram instalados geofones e/ou acelerômetros, na haste anterior a lâmina, que permitem as medidas de leituras de velocidade de ondas de compressão (Vp) e de ondas de cisalhamento (Vs). Este ensaio é similar ao downhole onde é analisado fisicamente o tempo gasto para determinado tipo de onda percorrer certa distância no interior da massa de solo.

O ensaio SDMT é executado com similaridade ao já consagrado ensaio DMT (Dilatômetro de Marchetti), só que

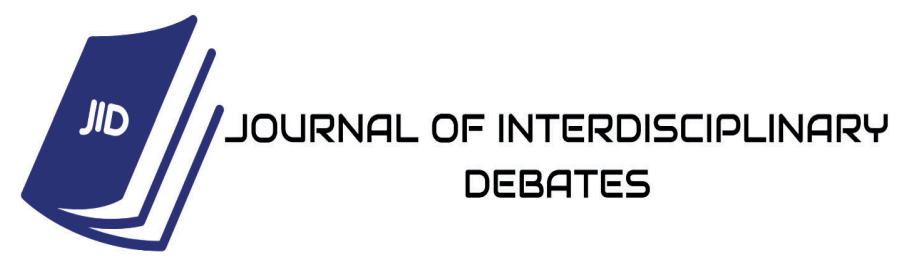


ISSN: 2675-469X

Vol. 02 - n 03 - ano 2021

Editora Acadêmica Periodicojs

em determinadas profundidades

tam as ondas produzidas por um

o ensaio é paralisado para se regolpe mecânico de martelo geragistrar as velocidades de propado na superfície. Detalhes desde gação da onda sísmica. Este reequipamento podem ser vistos na gistro é feito com a instalação de figura 1. dois sensores sísmicos que cap-
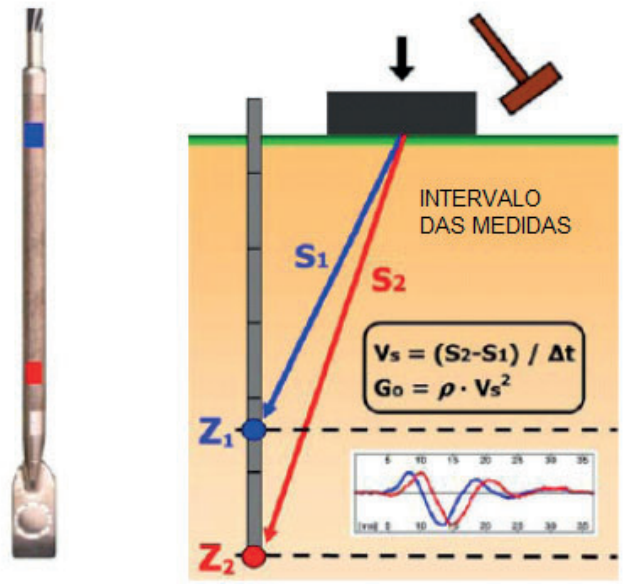

Figura 1 - Ilustração esquemática de funcionamento do SDMT.

Fonte: Adaptado de Foti et al. (2006).

Estes sensores normalmente são instalados com a distância entre si de um metro possibilitando determinar a diferença de tempo entre a emissão da onda e a respectiva captação de cada sensor. A diferença no intervalo de tempo é utilizada para determinação do módulo cisalhante à pequenas deformações do solo (Go). O ensaio SDMT é utilizado para fundamentar os conceitos teóricos de avaliação de cimentação propostos adiante. Estes conceitos incluem basicamente relações entre os parâmetros intermediários obtidos pelo ensaio SDMT e o módulo cisalhante à

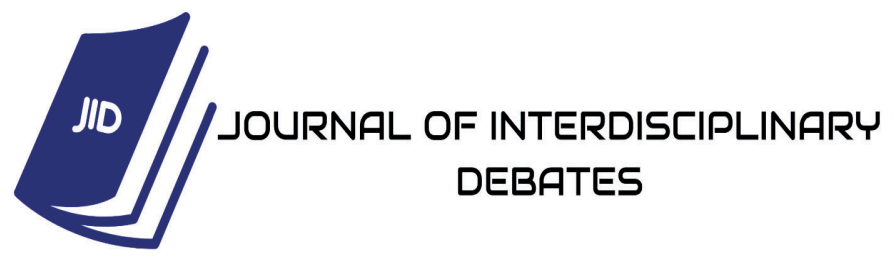


ISSN: 2675-469X

Vol. 02 - n 03 - ano 2021

Editora Acadêmica Periodicojs

pequenas deformações (Go).

Metodo da pesquisa

\section{Local da pesquisa}

A pesquisa foi realizada

em solos residuais originados do Complexo Águas Mornas do município de Santo Amaro da Imperatriz/SC. Maiores informações dos dados geológicos e pedológicos do local podem ser vistos em
Krueger (2015). O talude objeto da pesquisa é localizado na Rua Mansur Elias ao lado de um colégio. O local encontra-se a aproximadamente $60 \mathrm{~m}$ acima no nível do mar e o corte/talude tinha aproximadamente $22 \mathrm{~m}$ de altura com existência de 3 patamares executados como retaludamento (Figura 2)

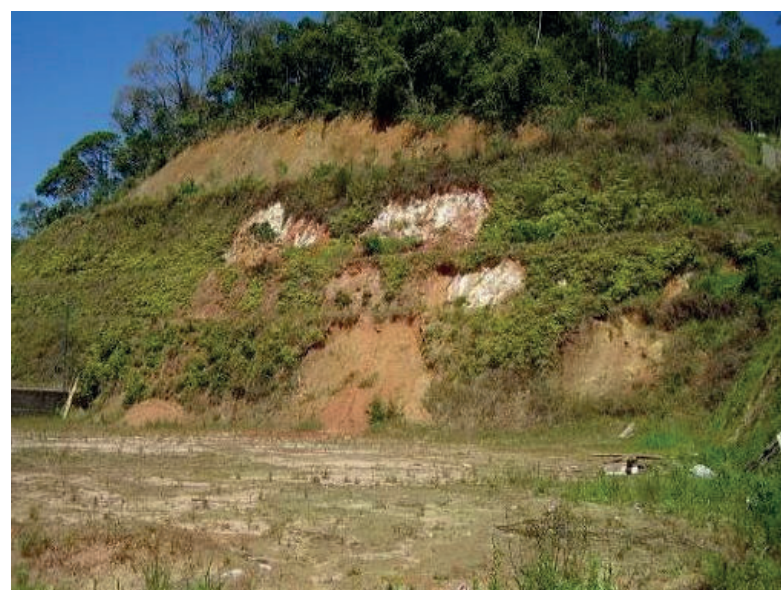

Figura 2 - Foto do talude

\section{Ensaios de Campo}

A pesquisa contemplou um procedimento de investiga- ção com ensaios de campo agrupados. Neste caso, foram conduzidos 2 grupos com 3 tipos de ensaios geotécnicos de campo

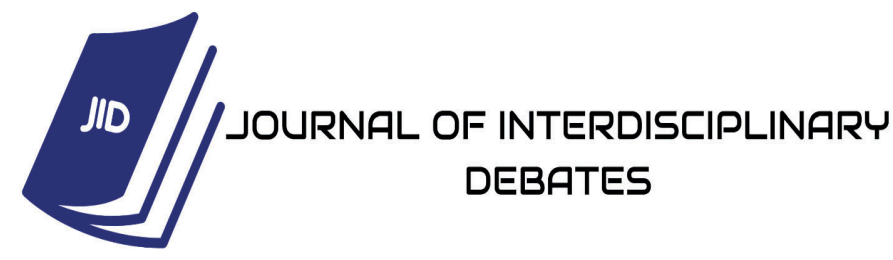


ISSN: 2675-469X

Vol. 02 - n 03 - ano 2021

Editora Acadêmica Periodicojs

(SPT, CPT e SDMT), em dois locais da área de pesquisa. O conhecido ensaio SPT permite obter o número de golpes (NSPT), o ensaio CPT registra a resistencia de ponta (qc) e o atrito lateral (fs). Os parâmetros intermediários obtidos pelo SDMT serão descritos a seguir. Estes ensaios foram conduzidos, respectivamente, conforme a NBR 6484, NBR 12069 e ASTM 6635.

Estes grupos foram alinhados de maneira a tornar a definição do perfil estratigráfico a mais fiel possível. Nesta metodologia as verticais são espaçadas de 1 metro em si o que permite uma comparação dos dados medidos ao longo da profundidade. No grupo 01 os ensaios atingiram uma profundidade de 12 metros, já no grupo 04, a cota de paralisação foi em 4 metros. Neste grupo, houve aumento rápido da resistência a penetração indicando um mataco ou a próvavel rocha matriz. Nesta situação a paralisaçãoo tem objetivo de preservar a integridade das ponteiras dos ensaios de campo. A locação dos grupos de ensaios de campo (furos) nos 01 e 04 pode ser visualizada em destaques na Figura 3.
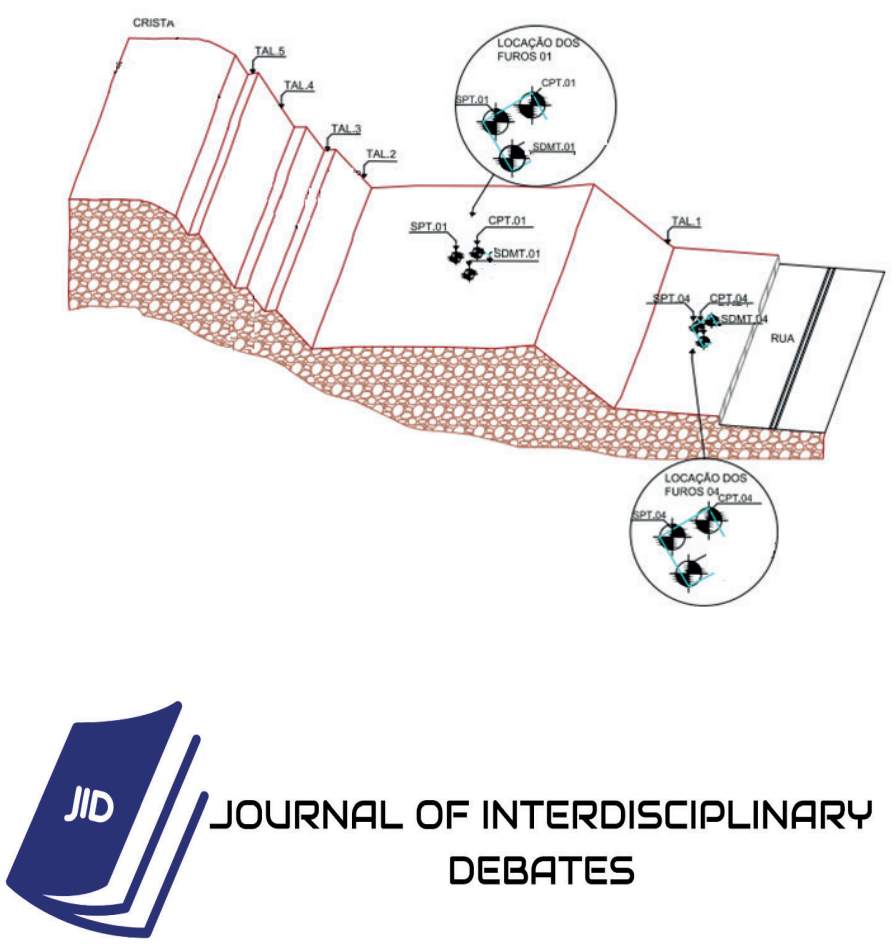
ISSN: $2675-469 X$

Vol. 02 - n 03- ano 2021

Editora Acadêmica Periodicojs

Figura 3 - Foto do talude e localização dos

grupos de ensaios de campo.

Fonte: Krueger (2015).

Condução dos ensaios de campo SDMT

O ensaio SDMT utilizou

o mesmo conjunto de hastes e equipamento de cravação do ensaio CPT. O ensaio foi realizado de acordo com norma americana ASTM 86 - "Método de ensaio padronizado para execução do DMT”. A membrana utilizada possui $0,25 \mathrm{~mm}$ de espessura e a calibração foi determinada de acordo com os critérios especificados por Marchetti (2001) com os respectivos acessórios de calibração que compõe o kit do ensaio. A pressão necessária para funcionamento do ensaio foi aplicada com a utilização de cilindro de ar comprimido. Durante o ensaio registrou-se as pressões po e pl (sem sistema automatizado de registro) que após processamento pelo software SDMTElab determinaram os parâmetros intermediários e, posteriormente, as propriedades geotécnicas ao longo da profundidade, em intervalos de $20 \mathrm{~cm}$. O ensaio SDMT confirmou a classificação do solo obtido pelos ensaios de campo SPT e CPT.

Como citado anteriormente, o ensaio sísmico SDMT possui dois acelerômetros nas hastes que permitem o registro da velocidade de onda gerada por um golpe de um martelo contra uma placa instalada na superfície do terreno. Nesta pesquisa o registro da velocidade de onda foi obtido a cada $60 \mathrm{~cm}$. Devido a dificuldade de acesso dos

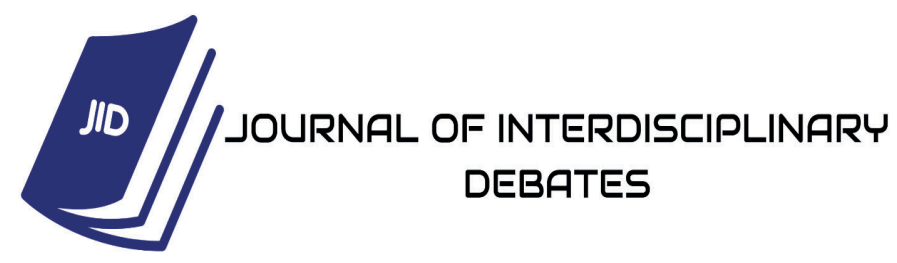


ISSN: $2675-469 \mathrm{X}$

Vol. 02 - n 03 - ano 2021

Editora Acadêmica Periodicojs

equipamentos a outros pontos do talude, os ensaios SDMT foram conduzidos na região dos agrupamentos de furos $\mathrm{n}^{\mathrm{o}} 01 \mathrm{e}^{\mathrm{o}} 04$. Detalhes da execução do ensaio sísmico podem ser vistos nas fotos nas Figuras 4a e 4b.

O nível de cimentação entre as partículas pode ser verificado através da análise da passagem de ondas sísmicas (Santamarina, 2001). A velocidade de onda sísmica é influenciada pelo tipo de contato existente entre as partículas do solo. Em solos fortemente cimentados tende a apresentar valores maiores do que em solos com baixo grau de cimentação.

Desta forma, a avaliação da variação de Go ao longo da profundidade permite acessar informações sobre a estrutura do solo. A utilização deste módulo normalizado em relação a resistência e deformabilidade tem sido bastante utilizada para definição de camadas dos solos, principalmente para definir os limites de solos cimentados, pouco cimentados e não cimentados. Para isso serão interpretados os valores da relação obtidas na divisão do módulo Go normalizado pelos índices NSPT, qc e ED, fazendo-se assim a correlação com os ensaios SPT, CPT e MDT respectivamente. Este tipo de interpretação tem sido feita por diversos autores como: Schnaid et al (2004), Viana da Fonseca et al. (2006), Giacheti et al (2006) e Cruz (2010).

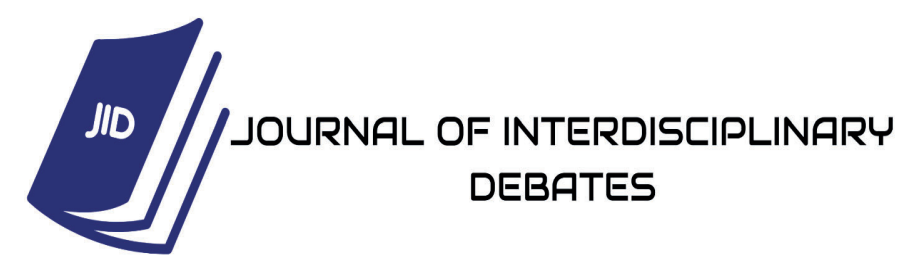


ISSN: 2675-469X

Vol. 02 - n 03 - ano 2021

Editora Acadêmica Periodicojs

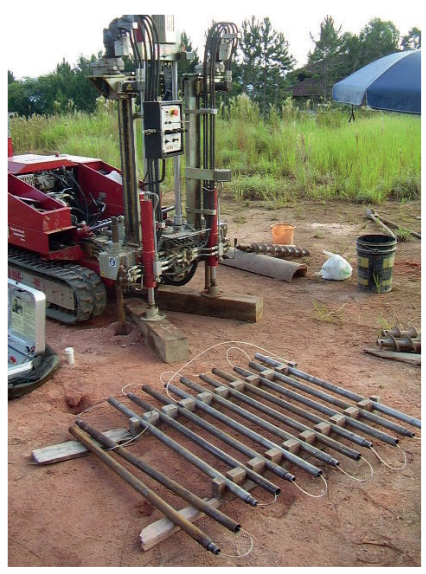

(a)

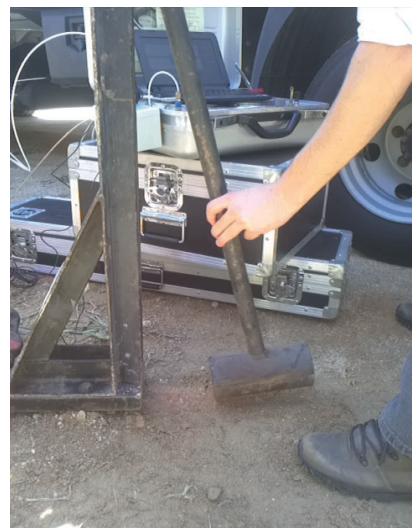

(b)

Figura 4 - Ensaio SDMT - (a) Preparação das hastes; (b) Golpe do martelo.

Fonte: Krueger (2015).

\section{Resultados obtidos}

Os ensaios SMDT conduzidos no local da pesquisa auxiliaram na classificação e confirmação do perfil de solo estimado pelo CPT e SPT O perfil estratigráfico via SDMT é obtido a partir do processamento das pressões po e p1 e, posterior determinação do índice de material (ID). A classificação do solo obtida pelo SDMT confirma que o material tem comportamento de silte arenoso à areia siltosa, mes-

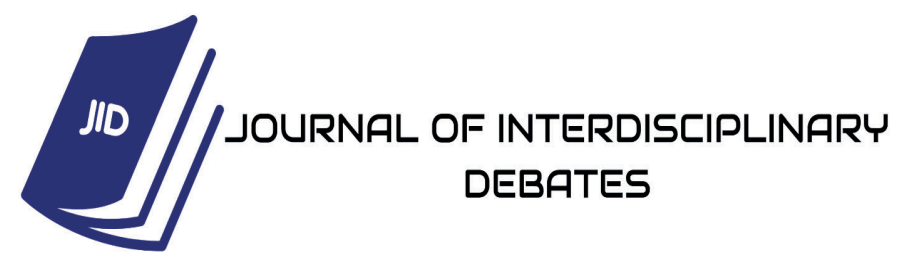


ISSN: $2675-469 X$

Vol. 02 - n 03 - ano 2021

Editora Acadêmica Periodicojs

ma classificação geotécnica obti- perfil de solo estudado pode ser

da pelos ensaios SPT e CPT. O visto na Figura 5.

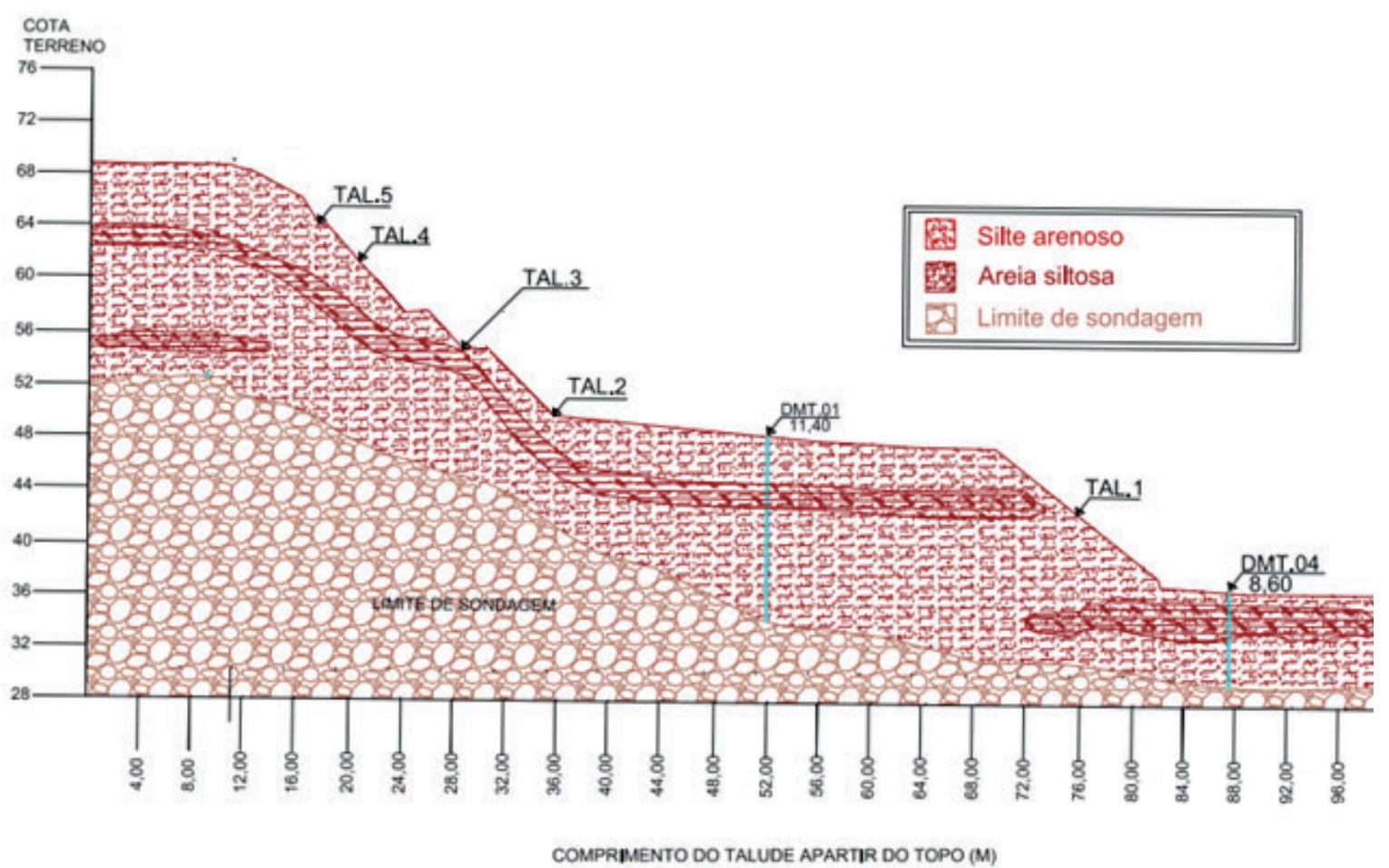

Figura 5 - Perfil estratigráfico obtido pelo

SDMT

Fonte: Krueger (2015).

Nas figuras 6 e 7, a se- horizontal (KD) e o módulo dilaguir, apresentam-se o perfil obtométrico (ED), para os dois grutido pelo ensaio SPT (à titulo pos de ensaios de campo. Nota-se de comparação) e os respectivos que os valores de ID confirmam registros do $\mathrm{N}$, os valores de qc, a classificação do material com os resultados das pressões corcomportamento silto-arenoso. Os rigidas (po e p1), o índice de valores de KD são usuais aprematerial (ID), índice de tensão sentando altos valores na superfí-

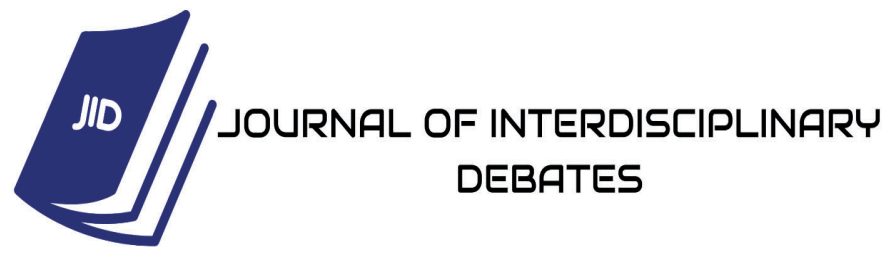


Vol. 02 - n 03 - ano 202

Editora Acadêmica Periodicoj

cie com tendência à diminuição e ou seja, quanto mais próximo da

estabilização ao longo da profun- rocha matriz, maior foi o valor do

didade. Já os valores de ED au- módulo dilatométrico.

mentaram com a profundidade,
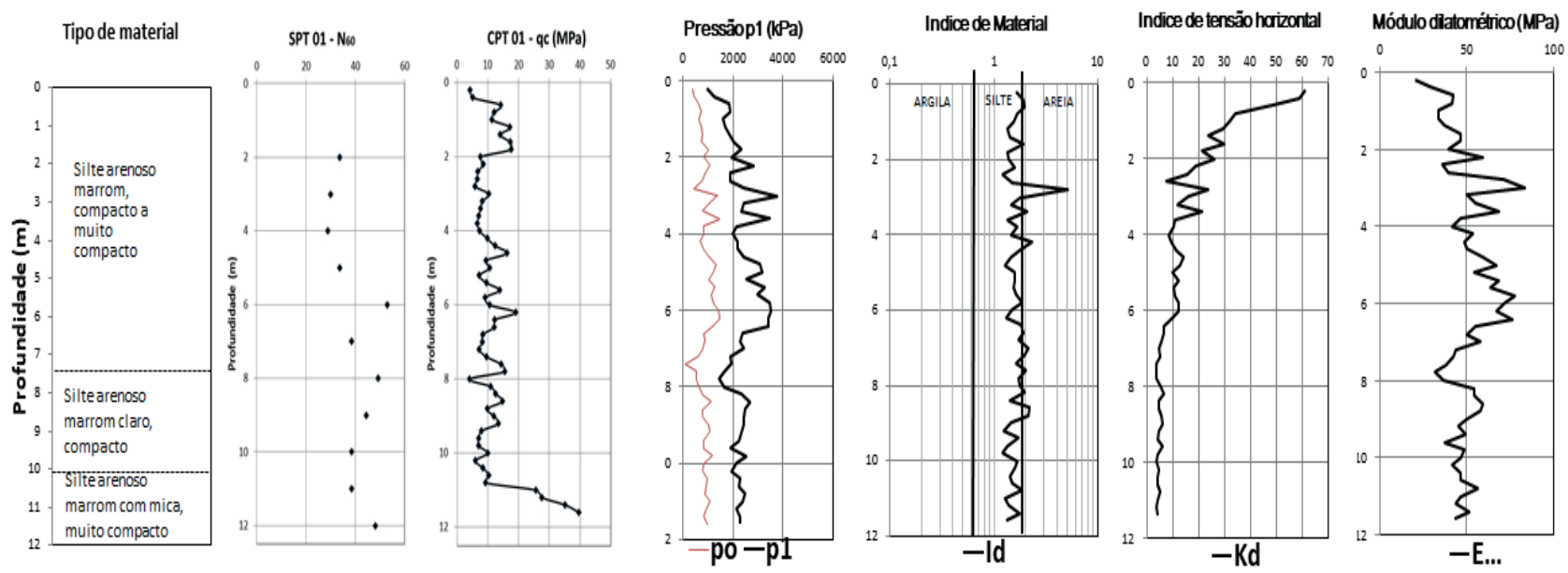

Figura 6 - Resultados do ensaio SDMT-01

Fonte: Autores.
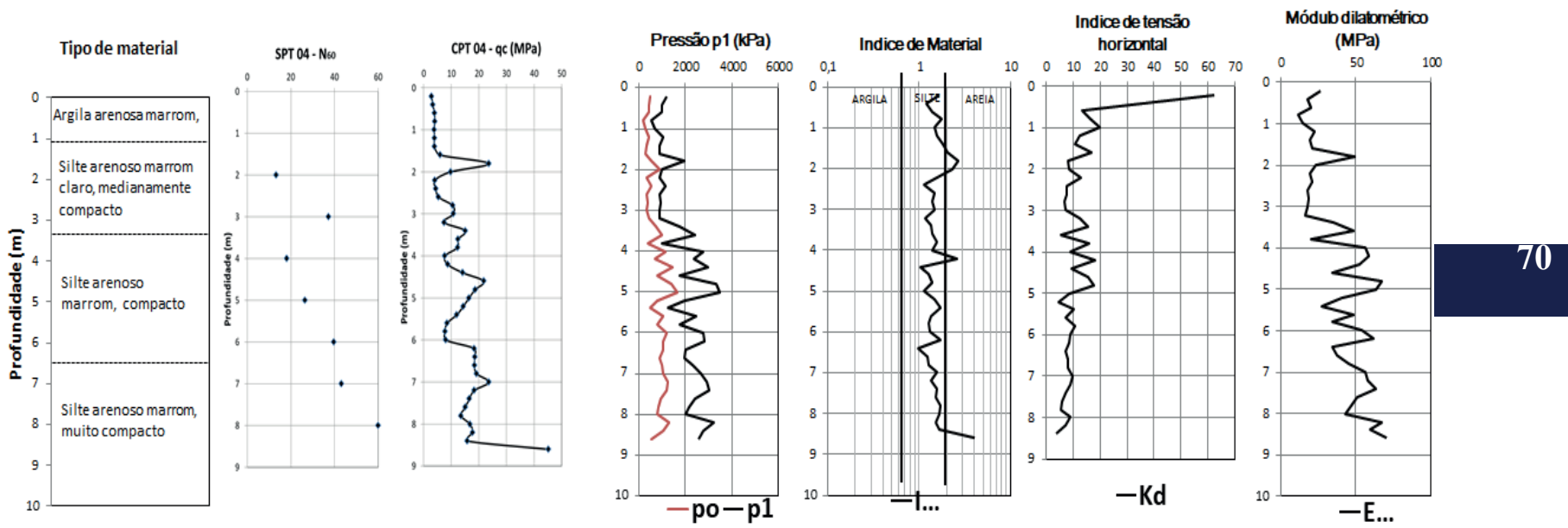

Figura 7 - Resultados do ensaio SDMT-04

Fonte: Autores.

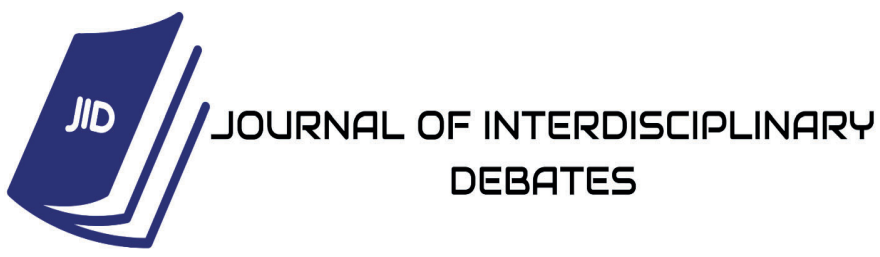


Os resultados da veloci- te podem ser visto a seguir na dade de onda e módulo cisalhan- $\quad$ Figura 8.
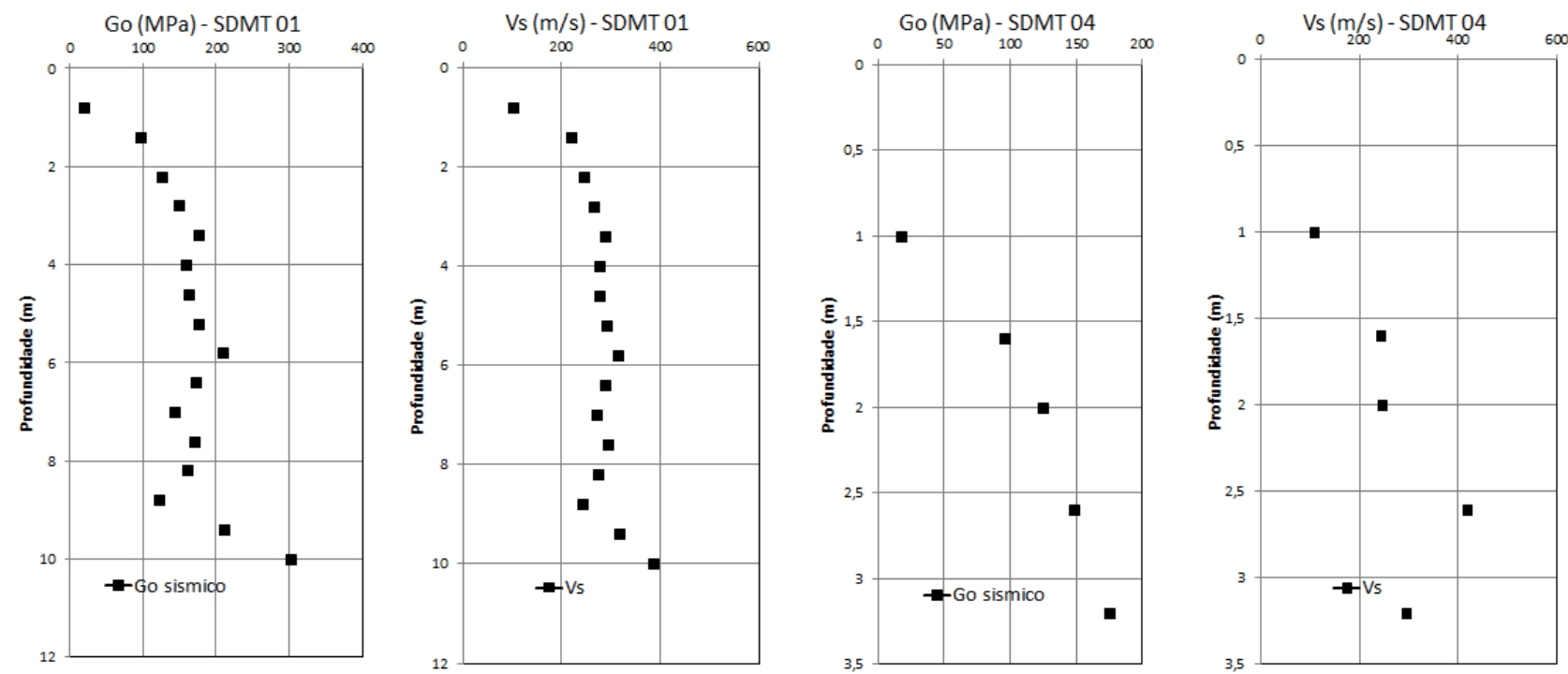

Figura 8 - Resultados de $\mathrm{V}_{\mathrm{S}}$ e $\mathrm{G}_{\mathrm{o}}$ nos SDMT-01 e SDMT-04.

Fonte: Autores.

Os ensaios do tipo -se que ocorre uma tendência de SDMT foram especialmente imaumento da velocidade de onda portantes para a determinação com o aumento da profundidadas velocidades da onda sísmide do ensaio. Num perfil de solo cas e do módulo cisalhante para residual à medida que se atinge pequenas deformações do solo camadas mais profundas são (Go), ao longo da profundidade. previsíveis que os níveis de ciA localização dos ensaios sísmimentação aumentem por se tracos realizados nos grupos 01 e 04 tar de materiais menos alterados. podem ser vistos nas Figuras 3 e 5. Ou seja, a estrutura e a ligação intergranular estão mais preNa Figura 8, percebe- servadas e isto contribui para o

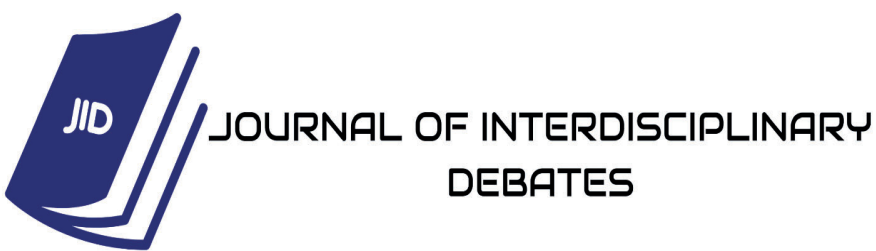


Vol. 02 - n 03 - ano 2021

Editora Acadêmica Periodicojs

aumento das propagações e das velocidades de ondas sísmicas. Nota-se que no furo SDMT-01, as velocidade de ondas (Vs) atingem valores aproximados de 210 $\mathrm{m} / \mathrm{s}$ na cota dos 6,0 metros, após tem uma leve queda no provável perfil saprolíto tendendo a atingir valores máximos de $300 \mathrm{~m} / \mathrm{s}$ na proximidade da rocha matriz. No furo SDMT-04, observa-se o mesmo comportamento, com aumento de VS com a profundidade e valores máximos (em torno de $400 \mathrm{~m} / \mathrm{s}$ ) antes da paralisação. Cabe salientar que a análise do grupo 04 ficou comprometida pela pouca profundidade atingida pelo SDMT-04. O mesmo atingiu um material resistente ou matacão durante sua cravação e teve que ser interrompido aos 4 metros.

Interpretando os resultados da Figura 8, nota-se que os valores de módulo Go au- mentam com a profundidade.

No furo SDMT-04 os valores de Go na profundidade aproximada de 3,0 metros é igual a $175 \mathrm{MPa}$. Confrontando-se que os registros realizados no SDMT-01 identifica-se um valor muito próximo de Go na mesma profundidade.

Devido a uma maior profundidade atingida no ensaio SDMT-01 pode-se fazer uma análise mais completa do comportamento de Go versus profundidade. Fica evidente que nas camadas superficiais ocorre um aumento de Go ao longo da profundidade, até o valor de $180 \mathrm{MPa}$. A partir desta profundidade, existe uma têndencia de estabilização e eventual queda (até 120MPa) nos valores de Go. Nas proximidades da paralisação (rocha matriz) observa-se um expressivo aumento dos valores do módulo sísmico, chegando a 300MPa.

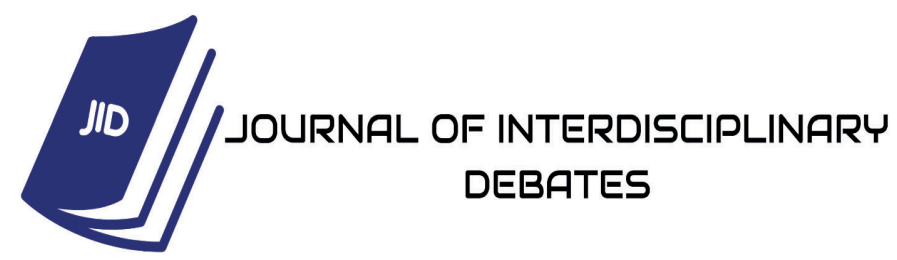


ISSN: $2675-469 X$

Vol. 02 - n 03 - ano 2021

Editora Acadêmica Periodicojs

Estes resultados cor- existe uma tendência de maiores

roboram com dados de outras valores de Go. Assim, relações pesquisas de campo que buscaentre módulo Go e outros indíram relacionar as propriedades de resistência e suas variações ao longo da profundidade. Os trabalhos de Robertson \& Campanella (1983), Viana da Fonseca et al. (2006) e Krueger (2015) mostraram que o ângulo de atrito de solos residuais oscila com a profundidade. Os autores obtiveram valores de ângulo de atrito altos nas camadas superficiais, os quais vinham a ter redução no manto intemperizado e, tornavam a aumentar na proximidade da rocha mãe.

Analisando-se os dados obtidos, entende-se que a cimentação natural tem uma parcela de contribuição na resistência total ao cisalhamento dos materiais. E que o módulo sísmico reflete este comportamento pois, quanto maior o nível de cimentação

Nas pesquisas de Krue-

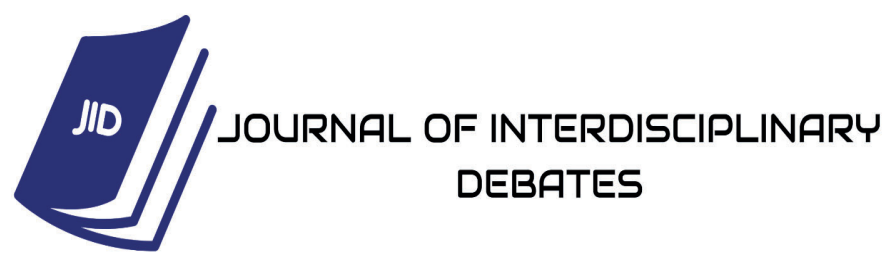


ISSN: 2675-469X

Vol. 02 - n 03 - ano 2021

Editora Acadêmica Periodicojs

ger (2015), no mesmo local, foram executados ensaios de compressão diametral em amostras cilíndricas submersas. O ensaio de compressão diametral fornece dados sobre a resistencia à tração, consequentemente, permite avaliar a estrutura intergranular. Este pesquisador escavou um poço de inspeção exatamente na área central do grupo des ensaios no 01 , desta pesquisa. $\mathrm{O}$ poço atingiu a profundiade de 3,0 metros. Para o ensaio de compressão diametral foram coletadas amostras indeformadas, a cada metro de profundidade. Durante a preparação do ensaio diametral, as amostras à $-1,0$ e $-2,0$ metros de profundidade desagregaram durante a submersão, dando indícios do baixo nível de cimentação neste solo. Os resultados dos corpos de prova na profundidade de $-3,0$ metros indicaram que os valores de resistência a tração variaram entre $1,92 \mathrm{kPa}$ e $6,15 \mathrm{kPa}$ (Krueger, 2015). Analisando os resultados do autor percebem-se baixos valores de resistência a tração indicando um baixo grau de cimentação nos solos residuais de gnaisse do local.

Realizando-se análise comparativa entre os dados sísmicos estimados em campo e resistência à tração determinada em laboratório podem-se obter algumas conclusões. Na figura 8 , notam-se o valore menores de Go e VS nas camadas mais superficiais do grupo 01. Concluindo-se assim que valores de Go inferiores a $150 \mathrm{MPa}$ e Vs menores que $250 \mathrm{~m} / \mathrm{s}$ tendem a indicar um baixo grau de cimentação das partículas de solos residuais de gnaisse.

No que refere a separação de horizontes com distintos níveis de cimentação empregou-se relações numéricas entre os

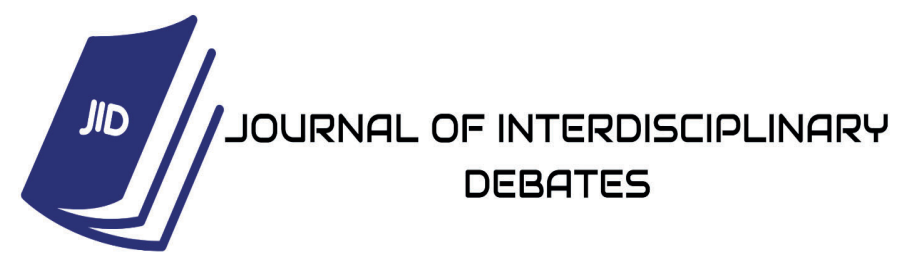


Vol. 02 - n 03 - ano 2021

Editora Acadêmica Periodicojs

ensaios de campo. Os resultados das relações Go/NSPT, Go/qc, Go/ED ao longo da profundidade para os grupos de ensaios 01 e 04 estão mostrados nas Figuras 9 e 10.

Como no ensaio SDMT os registros dos dados foi feito a cada $20 \mathrm{~cm}$ foi necessário realizar a média dos valores de NSPT, qc e ED no entorno da cota onde Go foi medido. O valor do NSPT utilizado nas análises refere-se ao valor de N60. Este é o índice de penetração utilizado internacionalmente e refere-se a uma eficiência de $60 \%$.
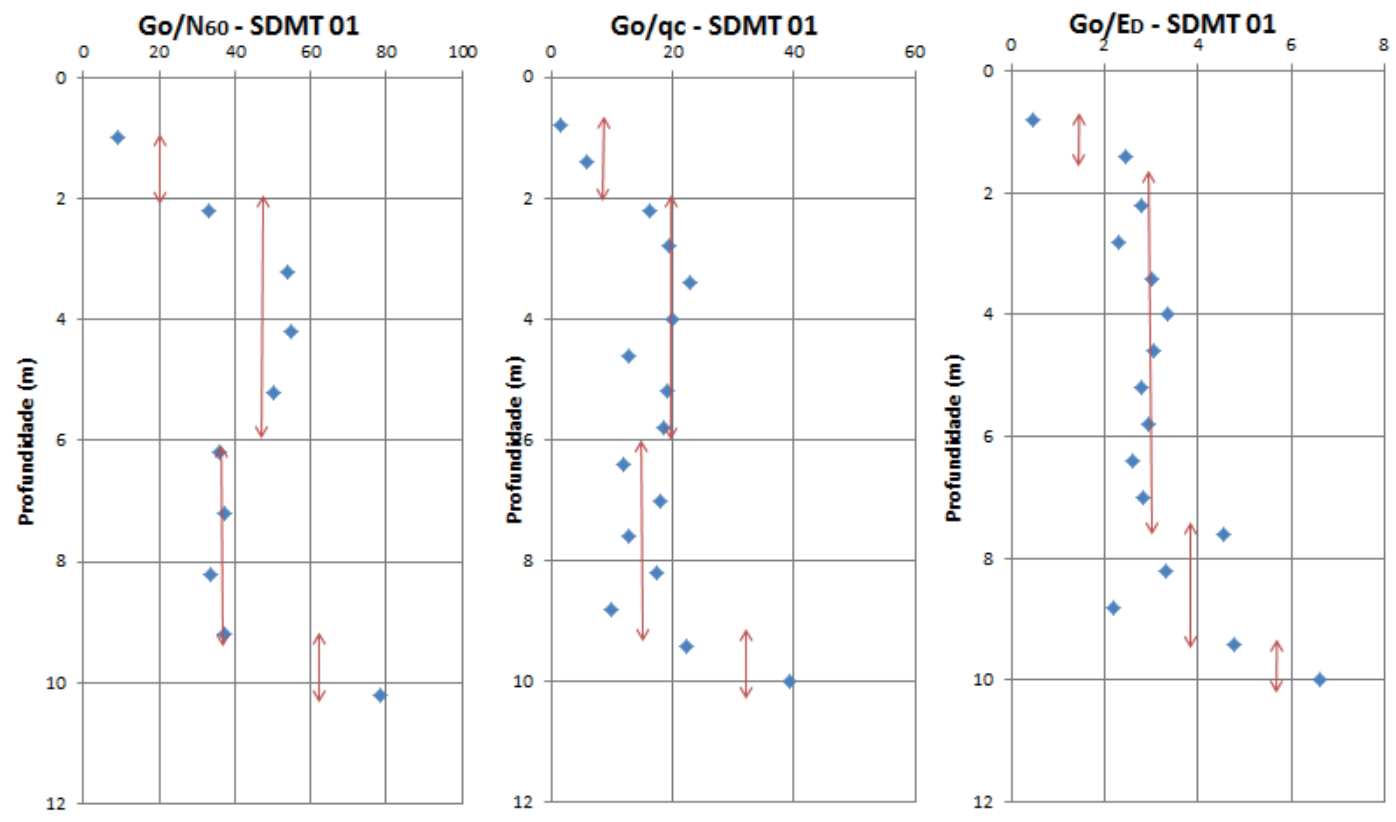

Figura 9 - Resultados dos ensaios de campo para identificação de cimentação no SDMT-01. Fonte: Autores. 
ISSN: 2675-469X

Vol. 02 - n 03 - ano 2021

Editora Acadêmica Periodicojs
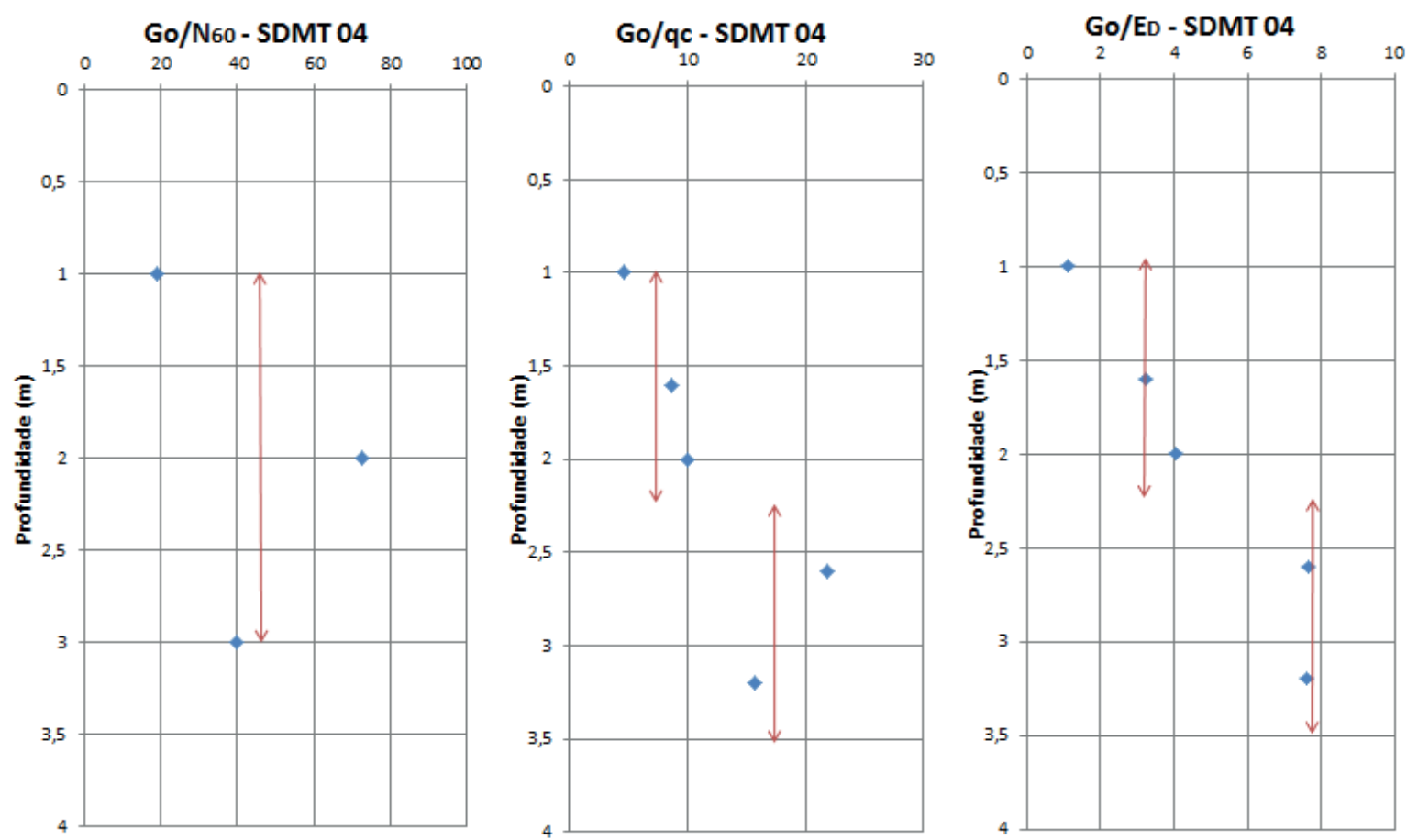

Figura 10 - Resultados dos ensaios de campo para identificação de cimentação no SDMT-04.

Fonte: Krueger (2015).

As relações entre o mó- de uma camada superficial até os dulo Go e os índices de resistên- 2 metros. Posteriormente, as racia NSPT e qc permitiram, prinzões Go/NSPT e Go/qc tendem cipalmente no grupo 01, definir a aumentar até os 6 metros indicamadas de solo com diferentes cando uma maior grau de cimenníveis de cimentação. Observantação e, diminuírem quando as do-se a Figura 9 (grupo 01), por relações são aplicadas em solos exemplo, verifica-se que os valomenos cimentados. A partir desres médios das relações obtidas ta profundidade houve aumenneste grupo (Go/N60, Go/qc, to da relação Go/NSPT e Go/qc Go/Ed) evidenciam a existência até atingir o impenetrável. Nesta 
ISSN: 2675-469X

Vol. 02 - n 03 - ano 2021

Editora Acadêmica Periodicojs

cota, para o grupo 01 , o valor médio de Go/NSPT é 60 e de Go/qc igual a 30 .

No grupo de ensaios 04 também parece existir a tendência da relação Go/NSPT e Go/qc apresentar menores valores, obtendo-se relaão de Go/NSPT em torno de 40 e de Go/qc igual a 15 , o que indica baixa cimentação.

Na avaliação da relação Go/ED para definição de horizontes lateríticos e saprolíticos, conforme trabalho de Giacheti (2006) e Schnaid (1998), esta relação apresentava valores maiores no horizonte laterítico e, decresciam conforme se aproximam do horizonte saprolítico, indicando um solo menos desenvolvido. Nos solos saprolíticos estudados por estes autores, quando bem próximo da rocha de origem, os valores de Go/ED eram inferiores a 10 .

Nesta pesquisa, os gru- pos 01 e 04 apresentam a relação Go/ED com valores médios de 3 nas camadas mais superficiais, tendendo a aumentar na proximidade do impenetrável, chegando a valores de Go/ED entre 5 e 8. O solo estudado é saprolítico, porque o horizonte laterítico foi escavada no retaludamento, assim não foi possível analisar a diminuição da relação Go/ED ao longo da profundidade. Porém, os valores médios da relação Go/ED ficaram em torno de 3 a 8 , confirmando baixos valores corroborando com os autores anteriormente citados.

\section{Conclusões}

De maneira análoga a outras pesquisas conduzidas em solos cimentados, a aplicação desta metodologia buscou definir limites para solos que possuem cimentações e limites onde estas 
ISSN: 2675-469X

Vol. 02 - n 03 - ano 2021

Editora Acadêmica Periodicojs

cimentações são muito baixas ou não existem. Além disso, indicou uma relação entre os módulos sísmicos e velocidade de ondas e o respectivo nível de cimentação intragranular.

Nesta pesquisa obteve um módulo sísmico máximo (GoMax) de 300MPa e VsMax de 400m/s. Numa análise comparativa, com ensaios de laboratório, que mostraram baixa cimentação no solo estudados os valores de Go foram inferiores a $150 \mathrm{MPa}$ e Vs menores que $250 \mathrm{~m} / \mathrm{s}$.

Nas correlações entre os ensaios de campo realizados os valores médios encontrados para Go/NSPT ficam entre 40 e 60 e para Go/qc oscilam entre 15 e 30. Na relação Go/ED ao longo da profundidade, os valores médios da relação Go/ED ficaram em torno de 3 a 8 , confirmando baixos valores de cimentação no solo estudado, corroborando com os autores anteriormente citados.

Conclui-se que o índice ED do dilatômetro é mais sensível para verificação da presença de cimentação do que KD. Pelo fato do registro de p1 ser obtido num solo menos perturbado pela penetração da sonda, a estrutura natural se encontra mais preservada refletindo mais diretamente em ED do que em KD. No que se refere a avaliação da cimentação através de ensaios sísmicos foram encontrados resultados satisfatórios que permitiram avaliar o nível de cimentação presente do solo deste trabalho. Todas as relações entre Go com índices do SPT(N60) do CPT(qc) e do DMT(Ed) mostram-se como boas alternativas para análise da microestrutura em solos residuais.

Evidenciou-se nesta pesquisa que, mesmo no horizonte saprolítico pode existir um 
ISSN: 2675-469X

Vol. 02 - n 03 - ano 2021

Editora Acadêmica Periodicojs

baixo nível de cimentação em solos residuais de gnaisse. Contudo deve-se entender que embora os ensaios tenham sido locados próximos e conduzidos com o mesmo cuidado e técnica a grande heterogeneidade macro e micro estrutural produz uma dispersão dos resultados.

\section{REFERÊNCIAS BIBLIO- GRÁFICAS}

\begin{abstract}
ABNT. Associação Brasileira de Normas Técnicas “NBR 12069 MB-3406: Solo - ensaio de penetração de cone in situ (CPT), CB02”. Rio de Janeiro, 1990.
\end{abstract}

ABNT. Associação Brasileira de Normas Técnicas. "NBR 6484: Sondagens de simples reconhecimento com SPT - método de ensaio, CB-02". Rio de Janeiro, 2001.
ASTM D6635. American Socie-

ty For Testing And Materials.

"Standart Test Method for perfoming the flat plate dilatometer." USA, 2015.

Cruz, N. "Modelling geomechanics of residual soils with DMT tests". Tese de Doutorado. Faculdade de Engenharia Universidade do Porto/Portugal, 2010.

Foti, S., Lancellotta, R., Marchetti, D., Monaco, P. And Totani, G. (2006). "Interpretation of SDMT Tests In a Transversely Isotropic Medium”. Proc. 2nd Intl. Conf. On The Flat Dilatometer, Wash. Dc: $275-280$.

Giacheti, H.L., Peixoto, A.S., De Mio, G., Carvalho, D. (2006). "Flat Dilatometer Testing In Brazilian Tropical Soils", Proceedings Second Flat Dilatometer Conference - Isc' 06, P. 103-111. 
Vol. 02 - n 03 - ano 2021

Editora Acadêmica Periodicojs

Washington/Usa.

Krueger, F.S. (2015). “Caracterização Física e Mecânica de Solo Residual de Gnaisse por meio de Ensaios de Campo e Laboratório - Santo Amaro da Imperatriz/ SC". Tese de Doutorado. Curso de Pós-Graduação Em Engenharia Civil. Universidade Federal de Santa Catarina. Florianópolis.

Leroueil, S. \& Vaughan, P.R. "The General and Congruent Effects of Structure in Natural Clays and Weak Rocks". Géotechnique, v.40, n.3, pp.467-488. 1990.

Maccarini, M. "Laboratory Studies of Weakly Bonded Artificial Soil". Thesis, University of London, 1987.

Mayne, P.W., Brown, D.A. (2003). "Site Characterization of Piedmont Residuum of North America". Characterization And Engineering Properties of Natural Soils, Vol. 2, Pp.1323-1339. Swets \& Zeitlinger, Lisse.

Schnaid, F. (2000). "Ensaios de Campo d Suas Aplicações à Engenharia de Fundações". Oficina E Textos. São Paulo.

Mitchell, J.K. "Fundamentals of Soil Behavior". 2nd ed., John Wiley \& Sons, New York, 1993.

Santamarina, J.C. 2001. "Soil Behavior at the Micro scale: Particle Forces." Proc. Symp. Soil Behavior and Soft Ground Construction, in honor of Charles C. Ladd - October 2001, MIT.

Schnaid, F., Fahey, M., Lehane, B.(2004). "In Situ Test Characterization of Unusual Geomaterial”. Geotechnical \& Geophysi- 
cal Site Characterization. Isc'2. Keynote Lecture. Viana Da Fonseca, A. And Mayne, P.W. (Eds). Milpress, Rotterdam, Pp.49-74.

Viana Da Fonseca, A., Carvalho, C., Ferreira, C., Santos, J. A., Almeida, F. Perreira, E., Feliciano, J., Grade, J. \& Oliveira, A. (2006). "Characterization of Profile of Residual Soil from Granite Combining Geological, Geophysical and Mechanical Testing Techniques". Geotechnical And Geophysical Engineering, 25, Pp. 1307-1348.

Wesley, L.D. (2010). “Geotechnical Engineering In Residual Soils". John Wiley \& Sons, Inc. Wroth, C. P. 1984. “The Interpretation Of In-Situ Soil Tests. Rankine Lecture.” Geotechnique, 4, 449-489. 